\title{
Correlation wind Lidar with an array detector and photon counting
}

Errico Armandillo, David Rees, Y. Tzeremes

Errico Armandillo, David Rees, Y. Tzeremes, "Correlation wind Lidar with an array detector and photon counting," Proc. SPIE 11852, International

Conference on Space Optics — ICSO 2020, 1185228 (11 June 2021); doi: $10.1117 / 12.2599360$

SPIE Event: International Conference on Space Optics - ICSO 2021, 2021, Online Only 


\section{International Conference on Space Optics-ICSO 2020}

Virtual Conference

30 March-2 April 2021

Edited by Bruno Cugny, Zoran Sodnik, and Nikos Karafolas
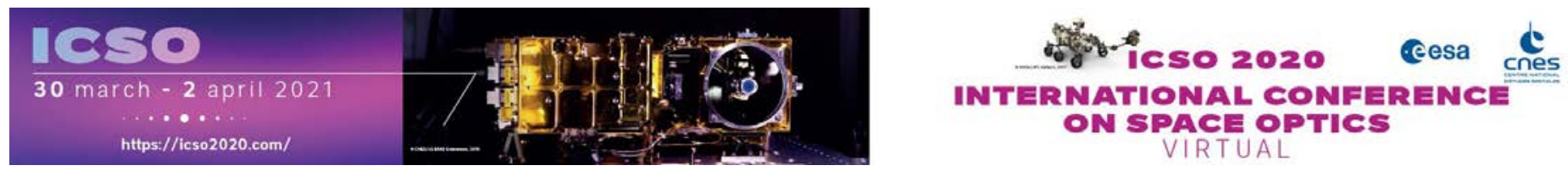

\section{Correlation wind Lidar with an array detector and photon counting}

\section{Cesa isoporecestings denes}




\title{
Correlation Wind Lidar with PMT Array Detector and Photon Counting
}

\author{
E. Armandillo ${ }^{\mathrm{a}}$, David Rees ${ }^{\mathrm{b}}$, Y. Tzeremes ${ }^{\mathrm{c}}$ \\ ${ }^{a}$ Eventech Ltd., 14 Dzerbenes St., Riga, LV-1006 Latvia \\ ${ }^{\mathrm{b}}$ The Paradigm Factor, UK \\ 'The European Space Agency, Estec, Noordwijk, The Netherlands
}

\begin{abstract}
We present the initial tests of a novel correlation wind lidar (CWL). The instrument scans the atmosphere and detects inhomogeneities in the aerosol content. Depending on whether it is being exploited from a ground-based, airborne or space platform, the instrument scans the atmosphere and by cross-correlation determines the viable structures for analysis and then determines wind from the motions of these viable structures. The current instrument exploits recent advances in photon-counting array detectors, advanced high-resolution time-tagging and image processing techniques. To improve the SNR of the system, a Rayleigh / Mie Spectral Separator is employed so that only the Mie signal from Aerosols is measured. In situations that are frequently encountered with low to modest aerosol content, this enhances the visibility of low-contrast aerosol structures, thus increasing the frequency and accuracy of wind determination. We are investigating the possibility of exploiting this on future Space Missions aimed at global wind measurement. While generally limited to the troposphere and tropopause regions, the technique does not require complex or sophisticated transmitter or receiver systems. Extension beyond Tropopause is proposed by complementing the CWL lidar with a Wind Doppler Interferometer operating in Limb Sounder on O2 lines. The CWL lidar concept can be extended to different applications domains.

Keywords: Lidar, Photon Counting, Signal Processing
\end{abstract}

\section{INTRODUCTION}

Knowledge of atmospheric winds is fundamental to Meteorology, Climatology, Atmospheric research as well as for Navigation. The wind techniques relevant to the work described here can be summarized in:

- $\quad$ Doppler lidar technique (DWL), as exploited by Aladin Doppler wind lidar on Aeolus satellite $^{1}$, launched by ESA August 2018. The first Satellite with a Payload of this kind ever flown.

- $\quad$ PBL (Planetary Boundary Layer) winds, where autocorrelation of local aerosols inhomogeneities provides winds 2,3

- Of historic interest: Atmospheric winds via Cloud Motion, as exploited by NOAA Satellites ${ }^{4}$, where correlation of Cloud images at different times provide wind.

The DWL technique of Aeolus is the most performant but also the most complex and challenging for both transmitter and receiver specifications. The transmitter laser operates in the UV @ $355 \mathrm{~nm}$ adequate energy, of around 100mJ, to ensure winds up the stratosphere. The requirements of spectral \& thermomechanical stability are very stringent \& demanding as well as the underlying issues of optical contamination \& damage are severe at such energy level in the UV and in vacuum. The receiver focal plane itself, also relies on tightly specified optics \& filtering components as well as durability \& stability in environment \& UV light. Last but not least, the associate data processing \& wind retrieval algorithm is matching the system in complexity \& susceptibility to errors \& bias.

The need for a simpler system has motivated this work. A wind lidar based on simplicity but also flexibility for an agile Lidar system whose basic concept can be adapted to a multitude of space applications: from a relatively larger system for winds, to smaller \& compact systems for altimetry, imaging, navigation, planetary mapping or landing assistance, etc. Basically, the concept proposed is based upon a scaling Tx, an array detector in photon counting, and a matched Data Processor whose Firmware, algorithm \& software can be adapted to the multitude of applications. 
Here we present a wind lidar concept able to provide vectors wind in the troposphere. The winds are extracted via crosscorrelation of Point Cloud Images generated by a scanning (for a ground systems), or a multi-line-of-sight (LOS), elastic backscattering lidar. For Space, such instrument can be complemented by a (passive) Doppler Wind Imager in Limb Sounding Mode detecting O2 lines to cover the altitude range: 10 to $30 \mathrm{~km}^{5}$. The elastic lidar operates on Mie, Aerosol scattering only while the uniformly distributed, molecular scattering is filtered out on the receiver by a Rayleigh filter. Motion of atmospheric aerosol produced by winds are then translated into 3-D local wind information via crosscorrelation of density variability in sequentially scanned or satellite moving atmospheric pixels. Cross-correlating all image pixels provide the required wind filed distribution. Hence Correlation Wind Lidar (CWL).

\section{THE CORRELATION WIND LIDAR CONCEPT}

The CWL lidar relies on a low-energy high repetition rate infrared or visible laser (but a UV laser is used in the demonstrator here discussed) sending beams of $\sim 0.1-1 \mathrm{~mJ}$ energy level @ 1-10 kHz repetition rate.

The receiver is based on a multichannel PMT detector array with single-photon-counting capabilities (in this study). The generated data sets, organized in matrices with indexes corresponding to pixel range $\&$ time, are then fed into a Processor performing cross-correlation between adjacent Matrices, A \& B. In this work the DSP (Digital Signal Processing) package of MATLAB is being used. In this case, maximizing the elements cross-correlation provides the correlation coefficients whose indexes values provide the position of the pixel, in space \& time, as it moves between A \& B. A formula does then provide the sought vector wind components.

Here the Transmitter requirements are very relaxed when compared to a DWL system: a multi-mode longitudinal laser beam with no particular frequency stability requirements, of relatively low energy \& high repetition rate is sufficient: AKA microlidar. So it is with the Receiver \& Processor: here photon counting capability of less than one photon per pulse is required and for the Processor, an adequate Storage Capability is needed to handle the large data set generated and needed for processing: storage capacity of approx. $1 \mathrm{~GB}$ is deemed adequate for the cases studies here. Fig. 1 gives a schematic of the CWL here studied, while Fig. 2 shows the concept for a wind space system.

\section{CORRELATION WIND LIDAR CONFIGURATION}

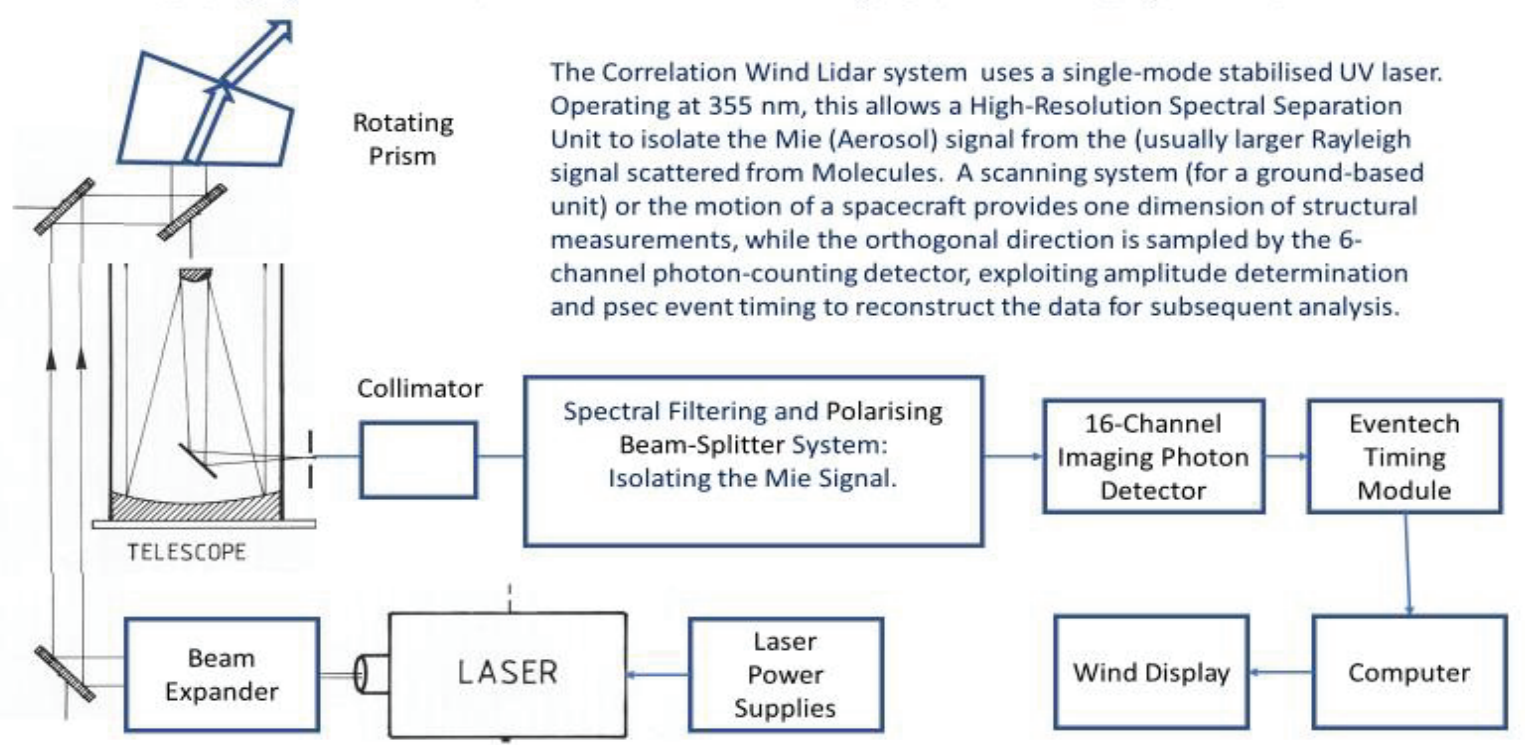


Figure 1. Schematics of the CWL instrument studied here: the transmitter laser scan the Atmosphere via an Azimuth scanning mirror. The Received Photons, are filtered of the Rayleigh components and detected/processed for wind acquisition.

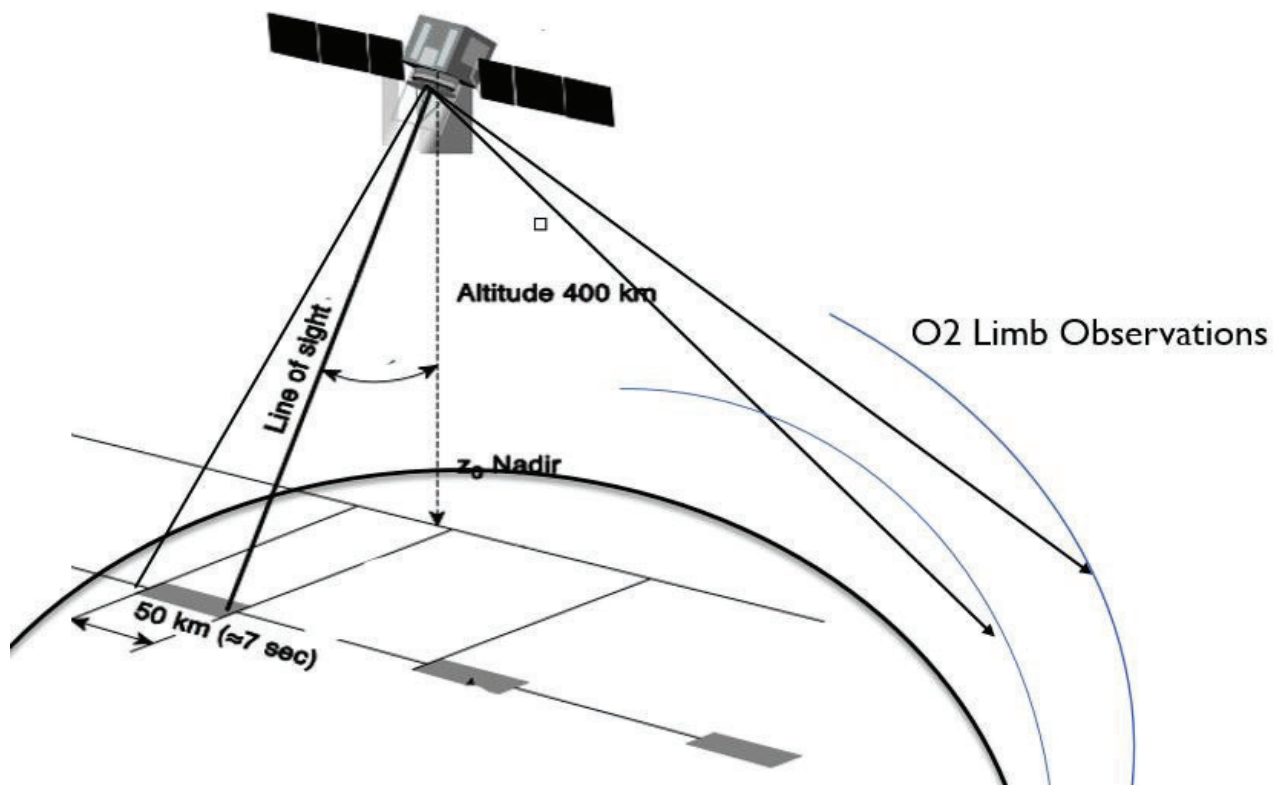

Figure 2. Schematics of the Wind Space concept. The Combo-Instrument satellite includes : the Multi line-Sight (two here) $\mathrm{CWL}+$ a Doppler Imager in Limb mode sounding the molecular Oxygen lines O2 in the altitude range 10-30 km (as HRDI in NOAA UARS wind Satellite)

Subsequent Figures 3, 4 \& 5 provide details of the actual CWL lidar system used in this work to demonstrate the CWL concept. The lidar is operated at Park Cottage Observing Station, located South London, West Sussex County. It is a rural area, subject to urban aerosols flowing out of London 6 .

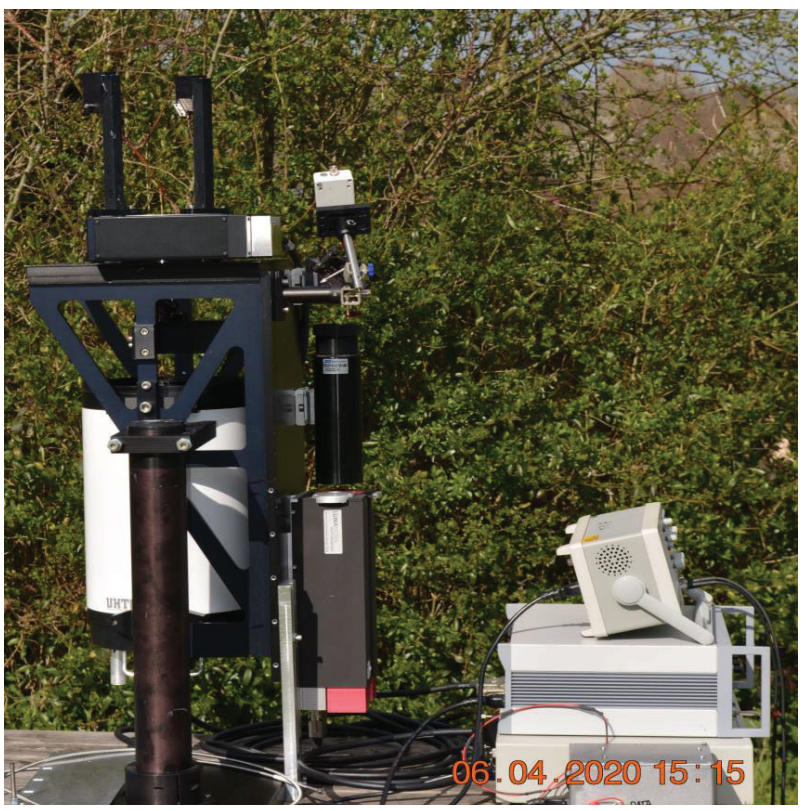

Figure 3. The CWL used in the experiments. It can be seen, $\mathrm{n}$ the left, the small transmitter attached to the lidar frame together with the receiver telescope. To the right, it is visible part of the receiver electronics. The laser transmitter is a commercial French-built TEEM laser, multi-mode, un-stabilized, providing o/p energy of $\sim 20 \mu \mathrm{J} @ 1 \mathrm{KHz} @ 355 \mathrm{~nm}$. On top of the laser it can be seen beam expander with a Newport Scanner \& mirror mount. 


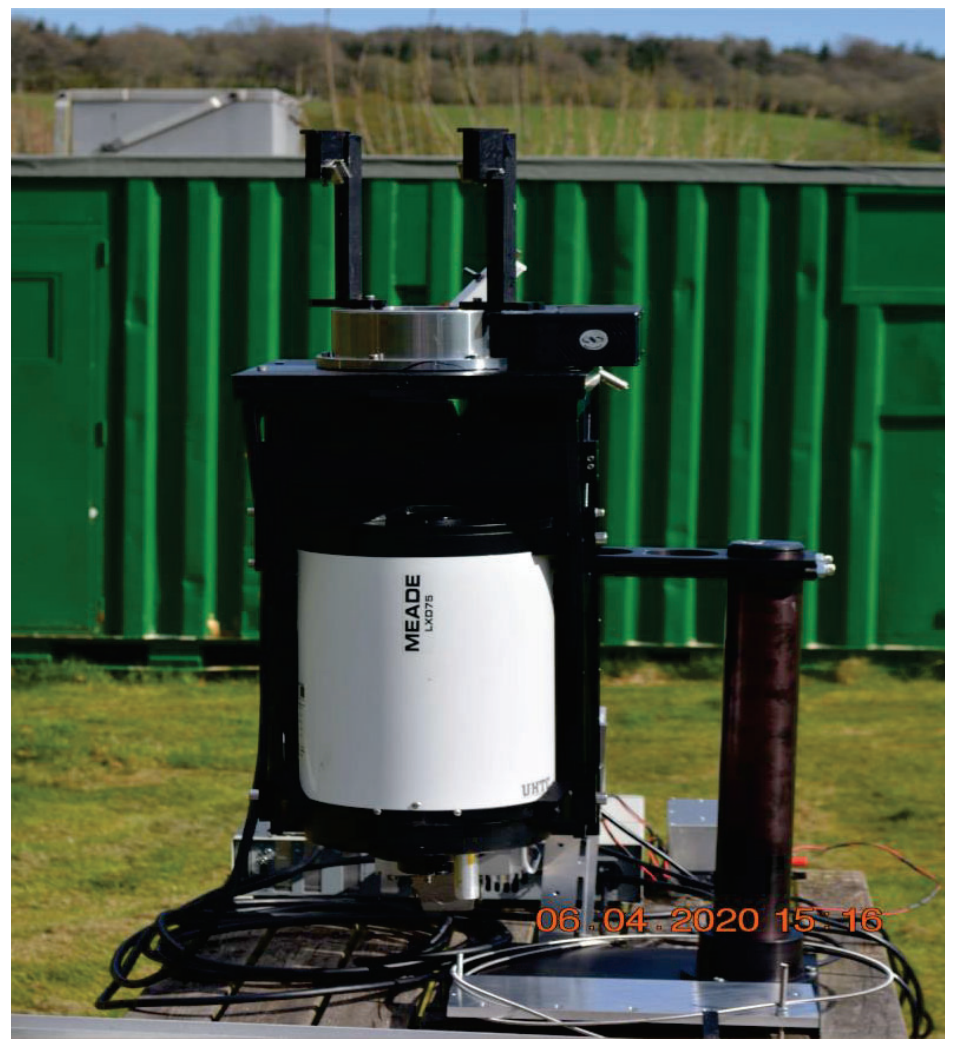

Figure 4. The figure shows the MEADE telescope, two 45 degree mirrors that turns the bistatic into a monostatic optical lidar configuration, the elliptical scanning mirror. Not present in this configuration is the bandpass \& Rayleigh filter. 

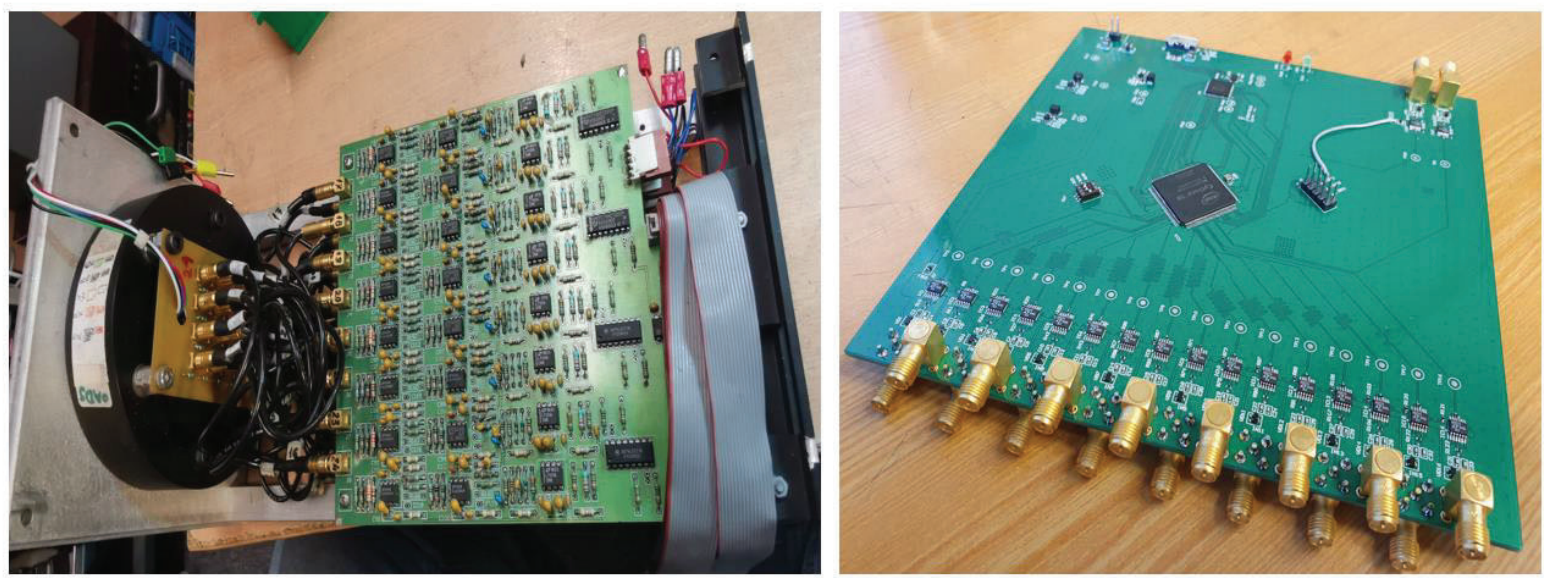

Figure 5. The figure shows on the left, a Photek 16 Channel array PMT-MCP array detector and electronics and, on the right, the 16 Channel Eventech single-photon-counting board electronics \& preprocessing

\section{CWL DATA SETS \& WIND PROCESSING: PRELIMINARY RESULTS}

The PMT-MCP detector output electronics provides signal intensity profiles versus height and azimuth angle (equivalent to time in this configuration). Data on-board pre-processing is first carried out and consists in: a) data acquisition from all laser pulses, b) pulse averaging, c) $\mathrm{R}^{2}$ multiplication \& normalization, d) background calculation \& subtraction. These data so generated are then used for wind processing. This is performed via NORMXCORR2 routine from MATLAB DSP package, but DSP also permits other functions, such as smoothing \& filtering, etc. Figure 6. shows an example of CWL data file as generated by the Point Cloud Data file and ready for processing.

\begin{tabular}{|c|c|c|c|c|c|c|c|c|c|c|c|c|c|c|c|c|c|c|c|c|c|c|}
\hline & & & & Real Time $\mathrm{H}$ & & & 0 & 30 & 60 & 90 & 120 & 150 & 180 & 210 & 240 & 270 & 300 & 330 & 360 & 390 & 420 & 450 \\
\hline & & & & $\begin{array}{l}\text { Background } \\
\text { Signal for } \\
\text { Profile }\end{array}$ & & & 143.695 & 129.512 & 129.961 & 130.078 & 129.875 & 129.84 & 130.956 & 129.818 & 130.342 & 129.508 & 129.438 & 128.601 & 127.573 & 128.104 & 104.77 & 57.775 \\
\hline $\begin{array}{l}\text { Summed } \\
\text { Signal/500 }\end{array}$ & Background & Corrected Signal & \begin{tabular}{l|} 
Real \\
Range V
\end{tabular} & $\begin{array}{ll}\text { Range } & \text { S } \\
\text { Squared } & \text { b }\end{array}$ & $\begin{array}{l}\text { Signal- } \\
\text { background }\end{array}$ & $\begin{array}{l}\text { Range } \\
\text { number }\end{array}$ & & & & & & & & & & & & & & & & \\
\hline & & & & & & & Channel 10 & Channel $1 \mathrm{C}$ & Channel $1 \mathrm{C}$ & channel $1 \mathrm{c}$ & annel 1 & Channel $1 \mathrm{C}$ & Channel $1 \mathrm{C}$ & Channel $1 \mathrm{C}$ & Channel $1 \mathrm{C}$ & Channel $1 \mathrm{C}$ & Channel $1 \mathrm{C}$ & Channel $1 \mathrm{C}$ & Channel $1 \mathrm{C}$ & Channel $1 \mathrm{C}$ & thannel $1 \mathrm{C}$ & thannel 1 s \\
\hline 26690.664 & & 101223262.5 & 50 & 2500 & 40489.305 & & 140633 & 30375 & 30247 & 30249 & 30456 & 29977 & 29876 & 30027 & 30381 & 30099 & 30256 & 30147 & 30137 & 30242 & 30457 & 30616 \\
\hline 26690.664 & & 145764000 & 60 & 3600 & 40490 & & 40633 & 30375 & 30247 & 30249 & 30456 & 29977 & 29876 & 30027 & 30381 & 30099 & 30256 & 30147 & 30137 & 30242 & 30457 & 30616 \\
\hline 22965.26 & & 41929300 & 70 & 4900 & 8557 & & 8700 & 1621 & 1525 & 1894 & 1561 & 1558 & 1734 & 1639 & 1388 & 1725 & 1722 & 1654 & 1761 & 1547 & 1829 & 1688 \\
\hline 49229.828 & & 41369600 & 80 & 6400 & 6464 & & 6607 & 65309 & 65303 & 65196 & 16 & 65261 & 65230 & 65357 & 65169 & 65191 & 65246 & 65228 & 65341 & 157 & 65401 & 65371 \\
\hline 56655.138 & & 29143800 & 90 & 8100 & 3598 & & 3741 & 62901 & 62609 & 62587 & 62658 & 62731 & 62477 & 62683 & 62648 & 62565 & 62536 & 62653 & 62757 & 62688 & 63092 & 62866 \\
\hline 52795.352 & & 2220000 & 100 & 10000 & 222 & & 365 & 59503 & 59451 & 59468 & 59117 & 59108 & 59374 & 59134 & 59218 & 58889 & 59157 & 59307 & 59672 & 59603 & 59695 & 59419 \\
\hline 48775.048 & & 746086000 & 110 & & 61660 & & 61803 & 55633 & 55443 & 55605 & 55126 & 55430 & 55463 & 55169 & 55159 & 55390 & 55292 & 55080 & 55772 & 55931 & 55708 & 55486 \\
\hline 44616.472 & & 823924800 & 120 & 14400 & 57217 & & 57360 & 51544 & 51385 & 51572 & 51122 & 51153 & 51189 & 51040 & 51092 & 50970 & 51006 & 50773 & 51465 & 51695 & 51177 & 51705 \\
\hline 40612.732 & & 888585100 & 130 & 16900 & 52579 & & 52722 & 47525 & 47451 & 46944 & 46987 & 46868 & 46859 & 46586 & 46641 & 46604 & 46577 & 46942 & 47500 & 47474 & 47450 & 47512 \\
\hline 36865.706 & & 8506400 & 140 & 19600 & 47934 & & 48077 & 43182 & 43225 & 42997 & 42989 & 42878 & 42807 & 42775 & 42772 & 42316 & 42608 & 42700 & 43219 & 43421 & 43340 & 43161 \\
\hline 33436.026 & & 981832500 & 150 & 22500 & 43637 & & 43780 & 39508 & 39234 & 39091 & 38916 & 38957 & 39078 & 38676 & 38954 & 38829 & 38469 & 38818 & 39451 & 39382 & 39377 & 39436 \\
\hline 30344.458 & & 1012582400 & 160 & 25600 & 39554 & & 39697 & 35979 & 35588 & 35609 & 35589 & 35196 & 35253 & 35343 & 35478 & 35241 & 35158 & 35544 & 35668 & 36066 & 35726 & 36088 \\
\hline 27542.692 & & 1041758300 & 170 & 28900 & 36047 & & 36190 & 32668 & 32220 & 32404 & 32038 & 32189 & 31978 & 32077 & 32443 & 31905 & 32092 & 32195 & 32507 & 32442 & 32719 & 32674 \\
\hline 25059.12 & & 1051023600 & 180 & 32400 & 32439 & & 32582 & 29534 & 29083 & 29374 & 29398 & 29091 & 29193 & 29210 & 29261 & 29068 & 29128 & 29209 & 29559 & 29727 & 29498 & 29470 \\
\hline 22837.98 & & 8711500 & 190 & 36100 & 29715 & & 29858 & 26791 & 26525 & 26699 & 26512 & 26560 & 26606 & 26567 & 26526 & 26537 & 26526 & 26515 & 27043 & 27157 & 27084 & 27074 \\
\hline 20866.02 & & 1081640000 & 200 & 40000 & 27041 & & 27184 & 24589 & 24113 & 24514 & 24169 & 23935 & 24070 & 24120 & 24120 & 24101 & 23949 & 24223 & 24432 & 24467 & 24623 & 24583 \\
\hline 19097.518 & & 198500 & 210 & & 24 & & 24728 & 22262 & 877 & 22134 & 21848 & 21950 & 22054 & 21878 & 21910 & 22068 & 21883 & 22037 & 22341 & 22384 & 22791 & 22389 \\
\hline 17532.874 & & 1090790800 & 220 & 48400 & 22537 & & 22680 & 20401 & 20287 & 20237 & 20189 & 20080 & 20142 & 20257 & 20103 & 20143 & 20262 & 20346 & 20701 & 20678 & 20679 & 20817 \\
\hline 16127.656 & & 188700 & 230 & 52900 & 20703 & & 20845 & 18784 & 18734 & 18755 & 18393 & 18444 & 18691 & 18544 & 18762 & 18578 & 18810 & 18784 & 19124 & 19093 & 19325 & 19128 \\
\hline 14868.952 & & 9491200 & 240 & 57600 & 19262 & & 19405 & 17419 & 17099 & 17203 & 17226 & 17093 & 17172 & 17230 & 17027 & 17365 & 17096 & 17452 & 17670 & 17595 & 17679 & 17581 \\
\hline 13735.504 & & 2187500 & 250 & 62500 & 17795 & & 17938 & 15893 & 15982 & 16031 & 15976 & 15720 & 15712 & 16044 & 16142 & 15875 & 15990 & 15952 & 16287 & 16291 & 16448 & 16501 \\
\hline 12719.914 & & 5525500 & 260 & 67600 & 16206 & & 16349 & 14623 & 14707 & 14785 & 14588 & 14827 & 14810 & 14817 & 14916 & 14862 & 14653 & 14684 & 14788 & 15087 & 15296 & 15212 \\
\hline 11801.664 & & 1099259100 & 270 & 72900 & 15079 & & 15222 & 13706 & 13766 & 13718 & 13757 & 13645 & 13705 & 13638 & 13643 & 13784 & 13703 & 13803 & 14146 & 14172 & 14184 & 14129 \\
\hline 10991.244 & & & 280 & & 14126 & & & 12748 & & & & & & 12744 & 12970 & 51 & 12645 & 12954 & 12973 & 13349 & 13257 & 13200 \\
\hline 10240.81 & & 1795400 & 290 & 84100 & 12994 & & 13137 & 12005 & 11991 & 11695 & 11803 & 12038 & 11827 & 11996 & 12064 & 12094 & 11987 & 12164 & 12201 & 12453 & 12321 & 12299 \\
\hline 9555.234 & & 7440000 & 300 & 90000 & 12416 & & 12559 & 11158 & 11048 & 11041 & 11128 & 11149 & 11026 & 11033 & 11241 & 11044 & 11005 & 11203 & 11460 & 11379 & 11634 & 11454 \\
\hline & & & 310 & 96100 & 11444 & & 11587 & 10461 & 10285 & 0473 & 10547 & 10395 & 10263 & 10347 & 10391 & 0488 & 0575 & 10389 & 10870 & 531 & 0716 & 0884 \\
\hline 8373895 & & $11155456 \mathrm{n}$ & ว२ก & 1"ח2רחก & 1 11894 & & $11 \cap 37$ & १६१९ & 9.8ก? & 9702 & Q६25 & 972.8 & १५२२ & १६7А & Q877 & $98 / 11$ & १९५ & १8:1 & 1กก72 & $102 n 1$ & 9987 & ก25ก \\
\hline
\end{tabular}

Figure 6. The figure shows CWL data files: either as xls (as shown) or csv format 
The CWL Lidar is typically operated at night to reduce solar background \& noises. The scanner run continuously and scan a complete rotation of 360 degrees in 33 seconds, at a constant elevation angle of 40 degrees.

For our Lidar system, Telescope overlap occurs at about 50 meters, so the data are being considered as from this height range. The range bin can vary from 5 to 10 meters and 1000 pulses are being integrated in each lidar channel profile, 4096 range bins are generated corresponding to $20 \mathrm{~km}$ to $40 \mathrm{~km}$ Data sets. The first two bins, out of 4096, are not used for the correlation, due to an artifact induced by the electronics. Fig. 7 below show pictorially the determination of the wind in Polar coordinates together with the formula used for the determination of the three vector wind components. The two concentric circles shown, correspond to two adjacent scans. In the example shown, the pixel (indicated as a star) moves outwards to the right due to the wind, which is calculated once the maximum of the correlation coefficient is known. The indexes $\mathrm{i}$ and $\mathrm{j}$, provide in fact the new range \& azimuth position due to wind. The arrows in Figure 7 indicate the correspondence between correlation matrix indexes and pixel displacement.

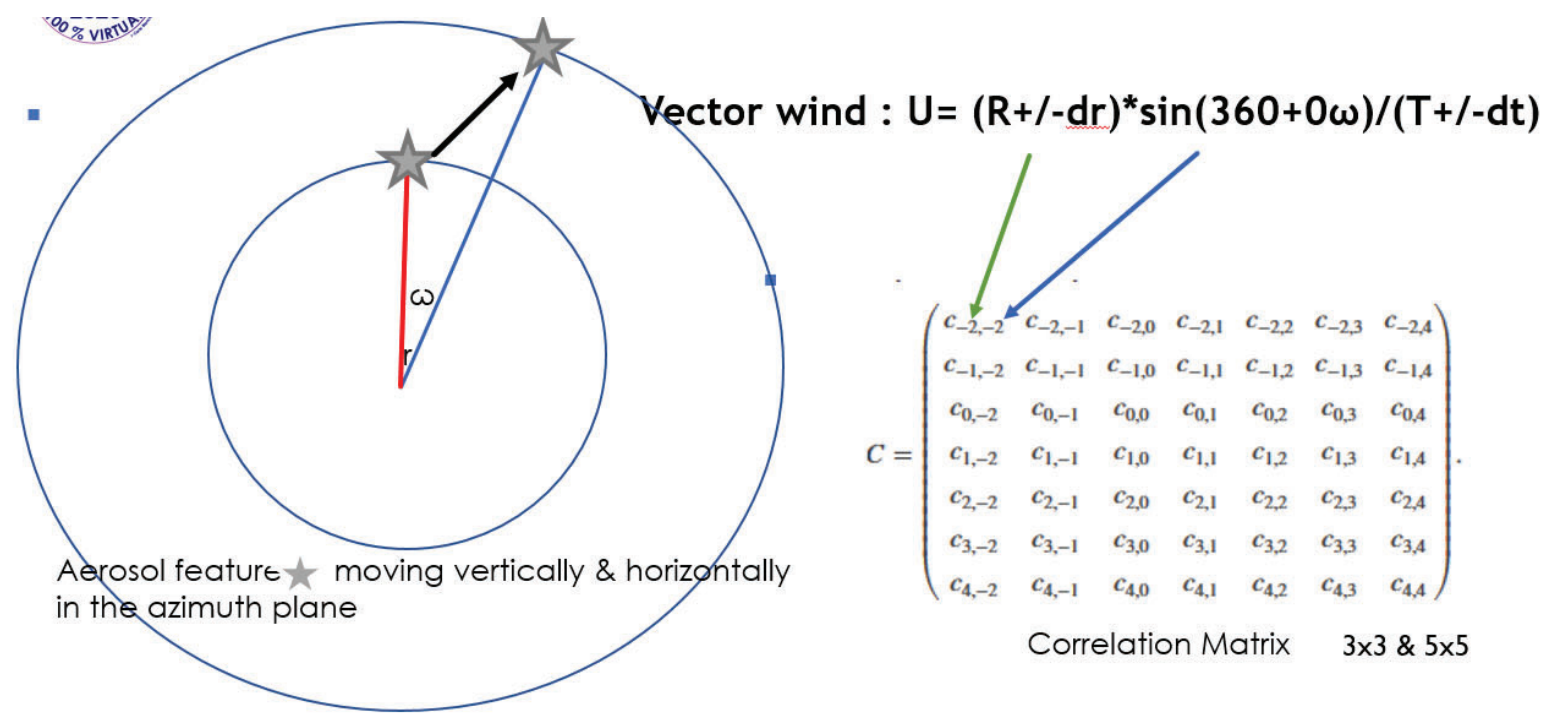

Figure 7. Pictorial view of wind vector calculation in CWL in polar coordinates. Left shown the motion of the aerosol feature moving with the wind during the scan and on the right it is shown the cross-correlation resulting Matrix with coefficient indexes providing the new coordinate position if the pixel.

Examples of wind calculations are now shown. Many Data sets were accumulated over the period May to November 2020. After this date the system went into refurbishment. In fact, after months of operation, the laser suffered UV optical degradation of the harmonic crystals which needed to be replaced. The energy had dropped to below $10 \mu \mathrm{J}$ as from September with the CWL still operating, but laser energy recovered to $25 \mu \mathrm{J}$ after repairs.

During the CWL runs over the May-November time frame, many data files were processed and atmospheric winds generated. Two show cases, run A \& run B, are presented as examples of CWL winds. Both cases refer to dry and clear weather conditions on May and September 2020 runs. In both cases visibility was good for a suburban London location with a clear sky and no apparent clouds.

In Figure 8, it can be seen that the max correlation coefficient follows exactly the lidar profile. After an initial saturation the correlation coefficient drops then raises again in correspondence to probably a light cloud or urban aerosol coming from London ${ }^{6}$. The saturation of the cross-correlation coefficient is associated also with a large scale azimuth invariance 
( $>100$ s meters), suggesting denser urban aerosols out from London. The Figure 9 gives the corresponding Point Cloud Image while Figure 10, provides the wind calculated for this set.

The Figure 11 provides the wind calculated at September run. These two cases are given as example of wind blowing with or opposite to the Scanner direction (positive or negative winds in the graphs).
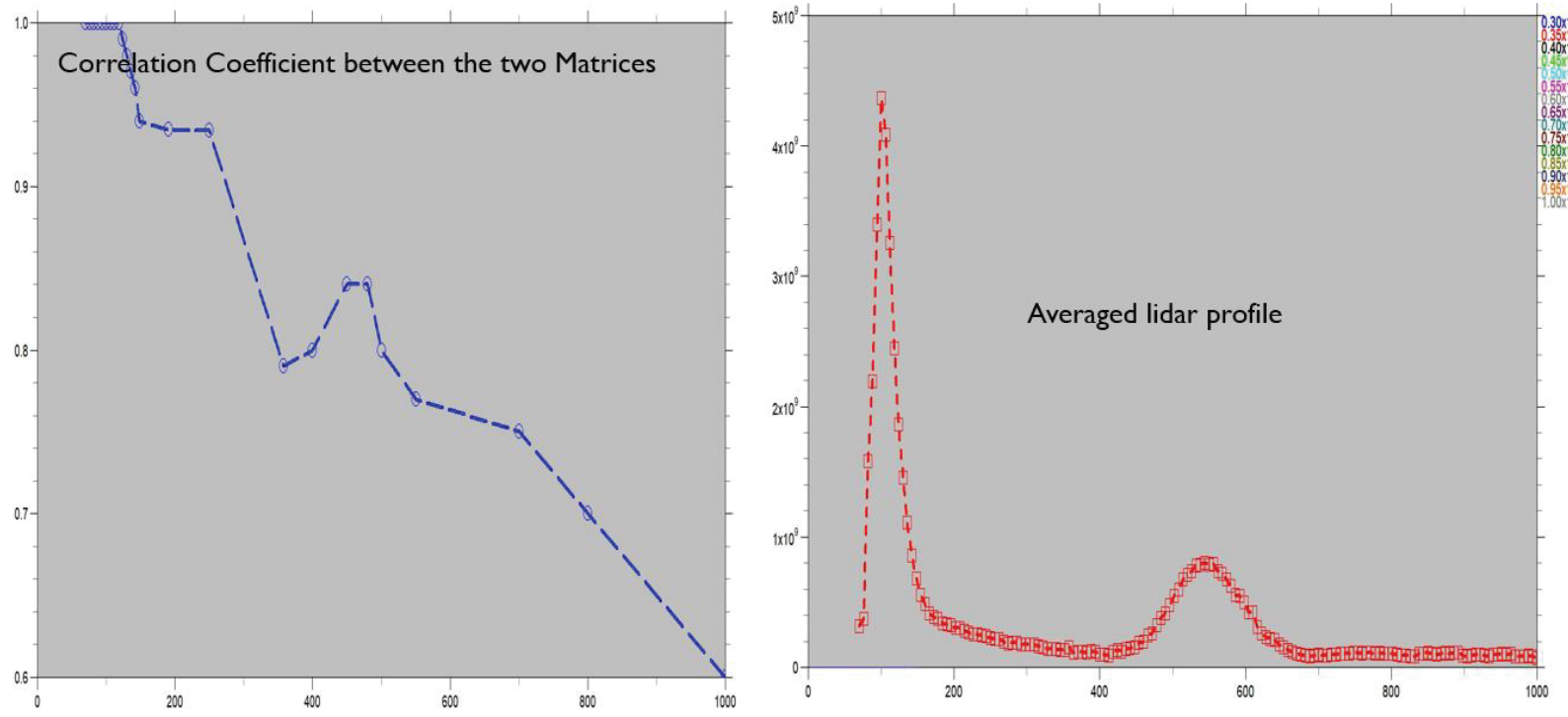

Figure 8. Picture shows the magnitude of the correlation coefficient (blue curve) corresponding to the lidar profile (red curve).
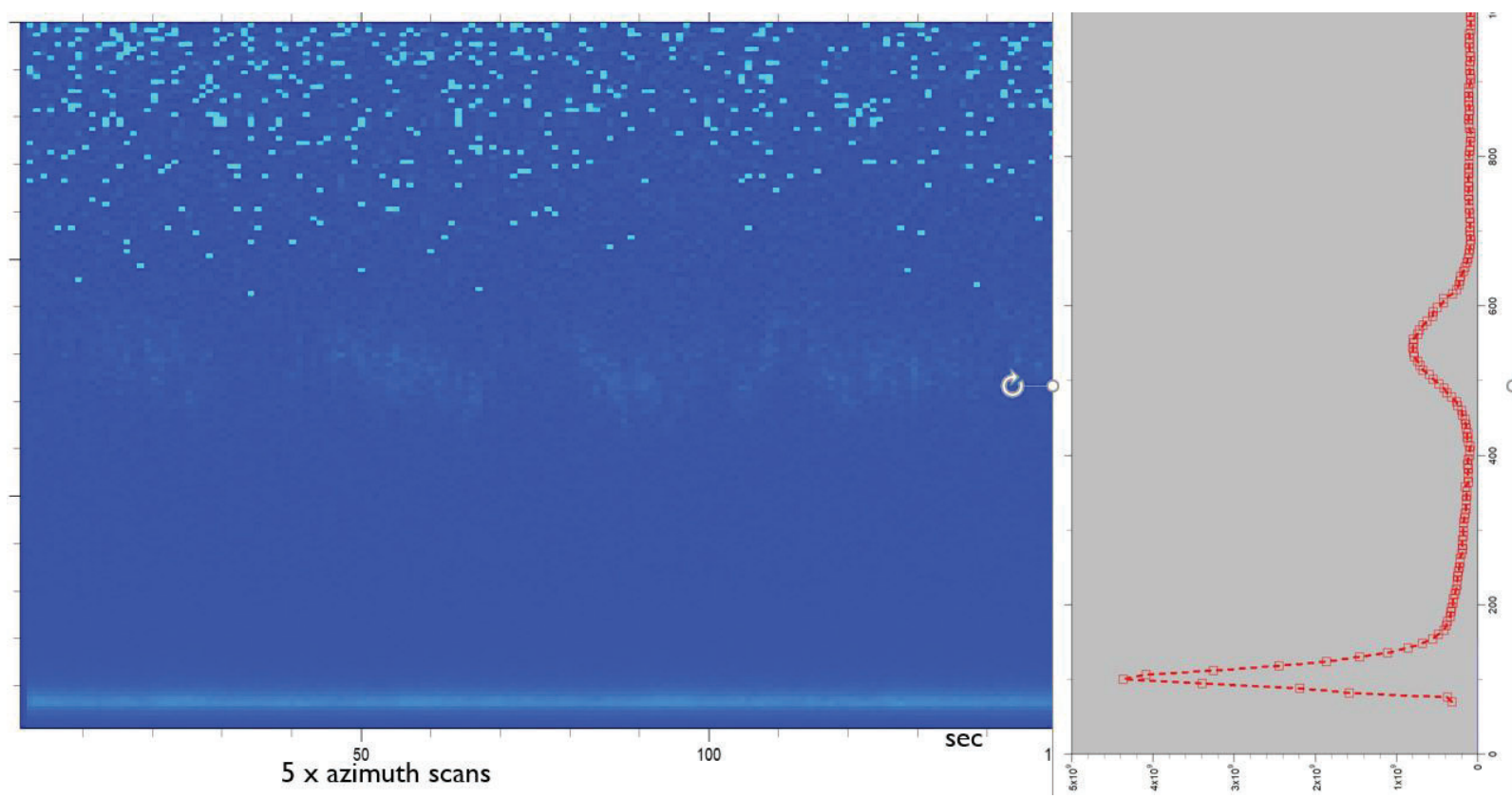

Figure 9. Picture shows the Point Cloud Image corresponding to above 

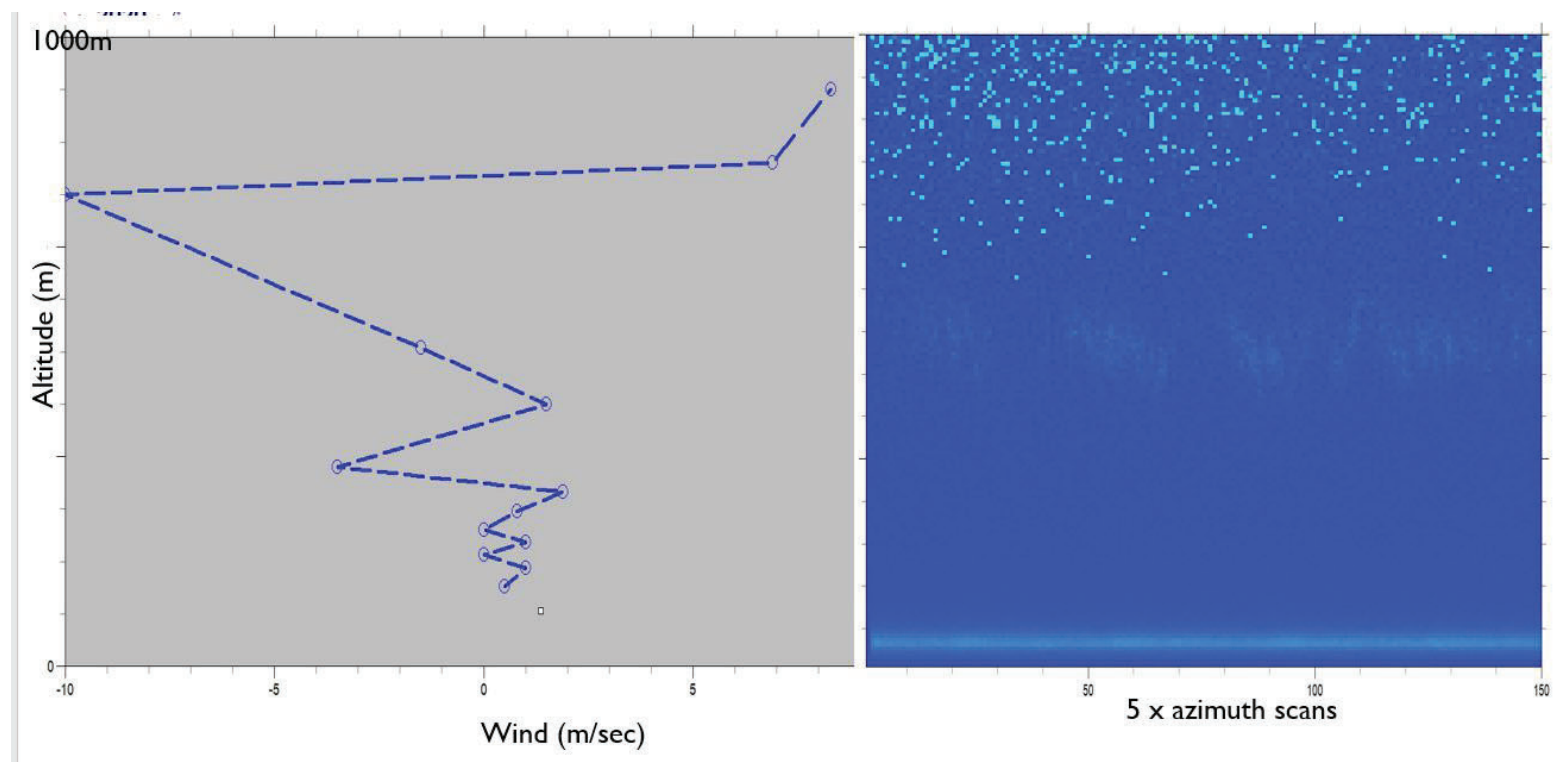

Figure 10. Picture shows the calculated vector magnitude of the vector wind, left with the corresponding atmosphere point cloud image. From the picture it can be seen that the wind runs opposite direction of the scanner.
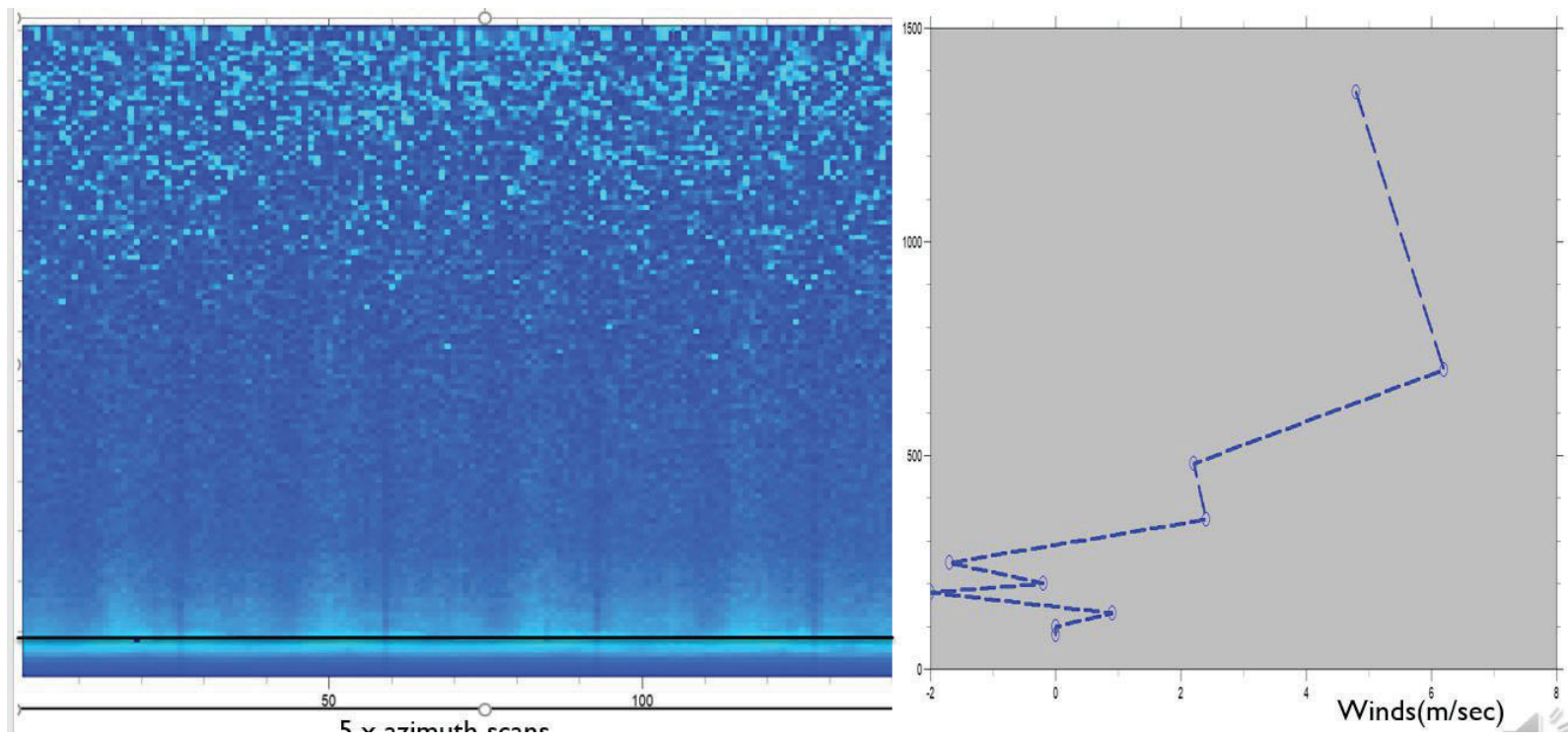

Figure 11. This run is shown for comparison to the wind in Figure 10, to show that in this case the wind blows in the same direction as the scanner rotation. 
To further understand the nature and the role of the scattering aerosols at low altitude and its relative azimuth invariance, an empiric methodology has been developed, and is being used to solve the lidar equation and calculate aerosol \& molecular extinction and scattering coefficients. This empiric method, based on actual lidar power profiles and local temperature \& pressure, will be reported in a future publication. In Figure 12 below it is plotted the calculated backscattering \& extinction coefficients for the run A (units are for backscattering: $\mathrm{m} . \mathrm{sr}^{-1}$ and for extinction: $\mathrm{m}^{-1}$ ). From the plots shown it can be seen that the values of backscattering coefficients versus height well match the values measured in Ref. 6 for this London sub-urban area.
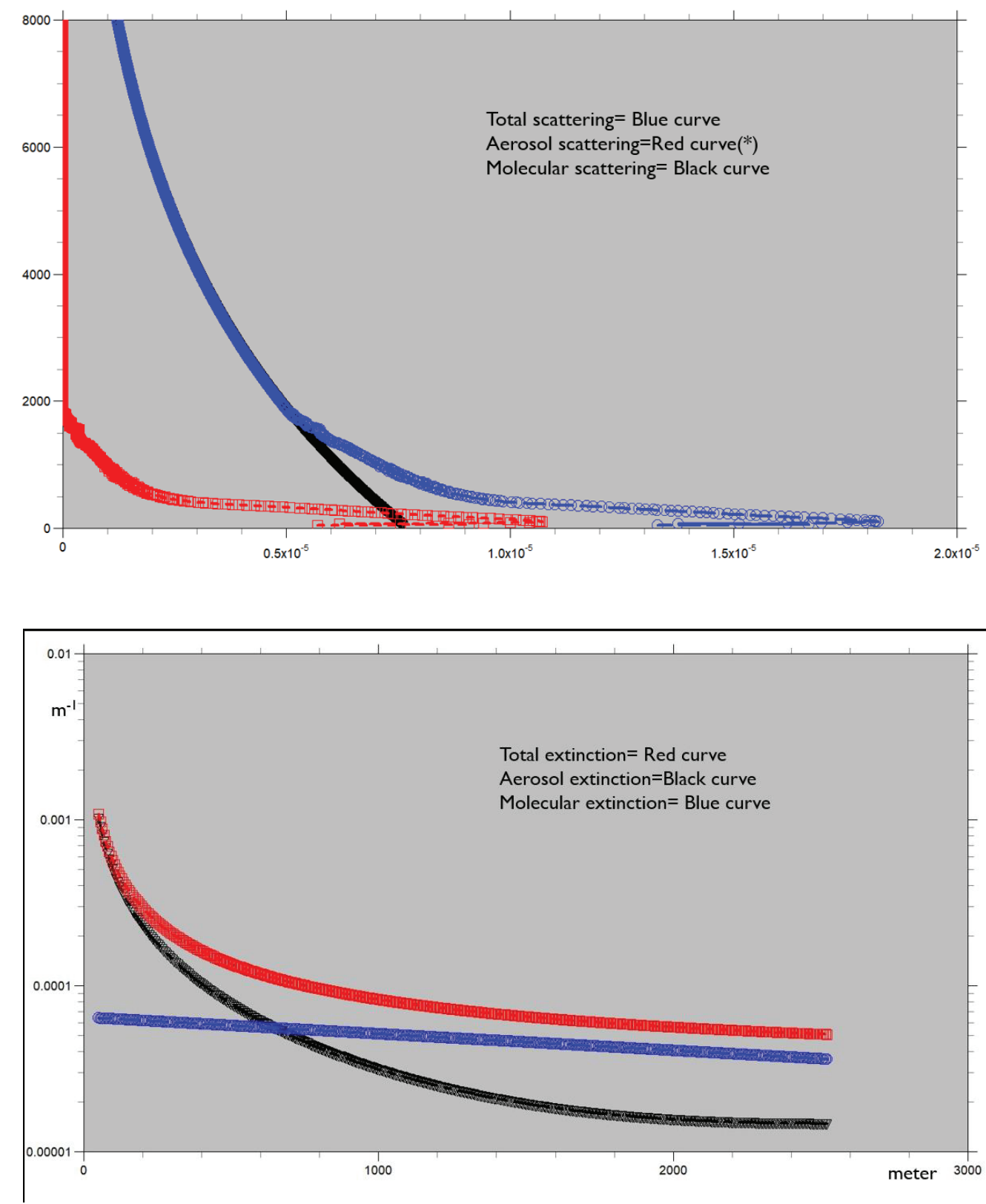

Figure 12. Calculated scattering \& extinction coefficients corresponding to Figure 8. 


\section{PRELIMINARY CONCLUSIONS \& ACKNOWLEDGMENT}

The CWL lidar technique with DSP processing has demonstrated that it can provide atmospheric winds, offering significant system simplifications in terms of Hardware, Software \& Processing than a DWL. These preliminary results show that further improvements on the present system concept can be obtained using a Rayleigh filter, to suppress molecular scattering, and the use of IR or Vis laser wavelengths to further reduce Molecular scattering. Although $\sim 10 \mu \mathrm{J} @ 1 \mathrm{kHz}$ level has provided good result, energies in the range of $\sim 25 \mu \mathrm{J}$ and/or higher repetition rate can significantly increase SNR and range.

For Space, a system study for the combination of CWL with a passive Doppler Imager in limb sounding is under consideration to fine tune a possible space wind instrument.

This work has been partially funded by ESA under PECS contract No. 4000130586/20/NL/SC.

\section{REFERENCES}

[1] https://earth.esa.int/web/eoportal/satellite-missions/a/adm-aeolus

[2] Kunkel, K.E., Eloranta, E.J., Weinman, A., "Remote Determination of Winds, Turbulence Spectra and Energy Dissipation Rates in the Boundary Layer from Lidar Measurements" J. Atm. Sciences, Vol. 37, 978-985 (1980)

[3] Mylapore ,A, Schwemmer, G, et al., "Proceedings SPIE, Baltimore, United States 2014"

[4] Leese, J. A, Novak, C.S., Clark, B.B., “J. Appl. Meteorology, Vol. 10, 118-125 (1971)

[5] Grass, H.J, Skinner, W.R, et al., “ Atmospheric Wind Measurements with the High-Resolution Dopler Imager”, J. of Spacecraft and Rockets, Vol. 32, 169-176 (1995)

[6] Ricketts, H., Vaughan G., Wareing D., “ Lidar Observation of Pollution Transport from London to Rural Areas", ILRC27, 2016 